\title{
Cross-layer network lifetime optimisation considering transmit and signal processing power in wireless sensor networks
}

\author{
Halil Yetgin, Kent Tsz Kan Cheung, Mohammed El-Hajjar, Lajos Hanzo

Q2 Abstract: Maintaining high energy efficiency is essential for increasing the lifetime of wireless sensor networks (WSNs), where the battery of the sensor nodes cannot be routinely replaced. Nevertheless, the energy budget of the WSN strictly relies on the 25 communication parameters, where the choice of both the transmit power as well as of the modulation and coding schemes (MCSs) plays a significant role in maximising the network lifetime (NL). In this study, the authors optimise the NL of WNSs by analysing the impact of the physical layer parameters as well as of the signal processing power (SPP) $P_{\mathrm{sp}}$ on the NL. They characterise the underlying trade-offs between the NL and bit error ratio (BER) performance for a predetermined set of target signal-to-interference-plus-noise ratio (SINR) values and for different MCSs using periodic transmit-time slot (TS) scheduling

30 in interference-limited WSNs. The NL maximisation is formulated as a non-linear optimisation problem taking into account the lower-bounded SINR, the energy consumption constraint, the maximum transmit power per link and again, periodic transmit-TS scheduling for all active TSs. The non-linear energy consumption constraint encountered is relaxed by employing a change of variables, which converts the problem into a linear form. Hence they can obtain the globally optimal solution of the original problem by solving a linear programming problem with the aid of the interior point method. The author's results

35 demonstrate that for a per-link target BER requirement (PLBR) of $10^{-3}$, a 'continuous-time' NL of 4.99 years (yr) is achieved by 1/2-rate convolutional coded soft-decoded quadrature phase-shift keying for an additive white Gaussian noise channel, when ignoring the SPP, which is reduced to $0.89 \mathrm{yr}$ because of the SPP. By contrast, the best NL that is achieved by 1/2-rate serially concatenated coding after ten iterations at a PLBR of $10^{-3}$ in a Rayleigh fading channel, which is reduced from 1.55 to 0.58 yr because of the SPP.

\section{Introduction}

Attaining a long network lifetime (NL) along with a low target bit error ratio (BER) is crucial in wireless sensor networks (WSNs), where regular battery replenishment can be impractical, or may even be futile in hostile environments. Therefore battery depletion imposed by the inappropriate choice of the communication parameters may have a major effect on the lifetime of a sensor node. However, the impact of the physical layer parameters on the $\mathrm{NL}$ is not explicitly characterised in the literature and there is only a paucity of contributions on the desired BER performance of the selected modulation and coding schemes (MCSs) as well as on its impact imposed on the NL in the context of WSNs. Explicitly, the choice of the system parameters has a vital effect on the BER, which in 60 turn has a considerable impact on the number of retransmissions that may result in a high energy consumption.

In [1], Chen et al. analysed the NL of a low-complexity physical layer relying on amplify-and-forward relays between a source node (SN) and a destination node (DN), where the BER performance was analysed in the low signal-to-noise ratio (SNR) regime. As detailed in [1], the relaying scheme between the transmitter and a receiver is capable of attaining a reduced-distance based path-loss gain and of conserving energy to prolong the NL, since the energy-dissipation (ED) imposed on the transmitting sensor was reduced with the aid of relays. The appropriate modulation mode capable of maintaining the target BER was discussed in [2], while maintaining a high energy efficiency, where on-off keying (OOK) and pulse-position modulation (PPM) was compared. The findings of [2] illustrate that the critical transmission range $d_{\mathrm{c}}$ can be readily found and that OOK is preferable to PPM for $d<d_{\mathrm{c}}$. The constellation size and transmission power selection strategy conceived for energy-efficient communications over fading channels was discussed in [3]. Rosas and Oberli [3] demonstrated that the careful selection of the optimal constellation size is capable of extending the NL by as much a factor of five. The authors of [3] also showed that as expected, binary phase-shift keying (BPSK) and quadrature phase-shift keying (QPSK) constitute the optimal choice for long transmission distances, while for short distances the optimal choice of constellation size may be either 16-ary quadrature amplitude modulation (QAM) or even 64-QAM. In [4], Cui et al. proposed a modulation 
scheme selection strategy for minimising the total energy consumption, including both the transmission and circuit energy consumption to deliver a predetermined number of bits. The authors of [4] demonstrated that optimised systems are capable of saving up to $80 \%$ energy compared to sub-optimum uncoded systems. The authors also observed that for coded systems, the impact of coding on the energy consumption depends both on the modulation scheme and the transmission range of the communication system. In addition, increasing the tolerable symbol error ratio of the physical layer facilitated energy savings because of the reduced transmit power [5], while there was an

145 increase in energy consumption because of the frame retransmissions. Plausible logic dictates that low-order modulation schemes like BPSK are energy efficient, unless achieving high data rates is necessary [6]. Nonetheless, the impact of the transmission rate on the NL is considered in [7], where an adaptive scheme is utilised for maximising the NL with the aid of a cross-layer design in interference-limited WSNs. In [8], the authors analysed the joint effect of the interference, power control and various forwarding strategies on the attainable NL. A similar study to ours was provided in [9], where the authors aimed for cooperatively maximising the minimum device lifetime for M-ary phase shift keying modulation under a specific BER constraint. An adaptive clustering technique maximising the NL, while maintaining the network's connectivity was

160 proposed in [10]. Similarly, as an application of WSNs, monitoring offshore wind farms was evaluated in [11], where a clustering protocol based on a three-level hierarchy and an algorithm jointly considering information routing, the instantaneous energy level of each node and the spatial distribution of the sensor nodes was proposed. Li et al. [12] conceived an ED model for a generalised turbo decoder architecture as a function of numerous design parameters, while characterising the trade-off between the ED of the signal processing power (SPP) $P_{\mathrm{sp}}$ and transmit power. For our model, we adopted the ED of the SPP relying on [12].

The BER is the salient quality of service metric of communication systems, but naturally, maintaining a longer NL for battery-limited devices is another important factor. Furthermore, the choice of the physical layer parameters, such as the MCSs, and transmit power substantially affects the NL and these parameters also have a direct impact on the BER. Therefore striking an attractive trade-off between the BER and the NL is crucial for network designers at an early design stage. Our new contribution is that we quantify the effect of the physical layer parameters on the achievable NL, while considering an application-dependent BER target in both line-of-sight (LOS) additive white Gaussian noise (AWGN) and in non-LOS Rayleigh block-fading channels in interference-limited WSNs. Moreover, we analyse the effect of the SPP on the NL.

The rest of this paper is organised as follows. In Section 2, we describe our system model and define the integration of adaptive MCSs into our system model. Then, our problem formulation and solutions are presented in Section 3, while our experimental results characterising a range of realistic application scenarios are discussed in Section 4. Finally, we conclude in Section 5.

\section{System model}

\subsection{Network model}

We consider $V$ half-duplex sensor nodes in the string topology of Fig. 1, where the $\mathrm{SN}$ is linearly connected to the DN by relay nodes (RNs). This string topology is exemplified for $V=10$ in Fig. 1. Given this simple network topology, $L=V-1=9$ denotes the number of links. Unidirectional communication is considered between the links and omni-directional antennas are used by each node. Each link has only a single intended receiver. We denote the total number of time slots (TSs) per TDMA frame by $N$.

\subsection{Channel and MAC layer model}

Each node can only transmit or receive in TS $n$. Owing to the half-duplex nature of the transceivers, a receiver can only communicate with a transmitter over the shared wireless channel within the same TS. The channel gain of a link between the transmitter $i \in\{1, \ldots, V\}$ and receiver $j \in\{1, \ldots, V\}$ is given by $G_{i, j}=1 /\left(d_{i, j}\right)^{m}$, where $d_{i, j}$ is the geographic distance between the nodes $i$ and $j$, while $m$ is the path-loss exponent. Naturally, node $i$ can only transmit at a limited power, which is lower than the maximum transmit power assigned to that node. Each node has an initial battery energy that cannot be exceeded by the total ED of that node.

The LOS AWGN channel is defined by a certain propagation path-loss model and a fixed noise power at the receivers. The link quality is defined in terms of the signal-to-interference-plus-noise ratio (SINR), which is denoted by $\Gamma_{l}$ in the AWGN channel model and given by Goldsmith [13] $\Gamma_{l_{i, j}, n}=\left(\left(G_{i, j} P_{i, n}\right) /\left(\sum_{i^{\prime} \neq i} G_{i^{\prime}, j} P_{i^{\prime}, n}+N_{0}\right)\right)$ for a specific link $l$, where $P_{i, n}$ denotes the transmit power of node $i$ in TS $n$ and $N_{0}$ is the noise power at the receiver.

In addition, for fading scenarios we assume a Rayleigh fading channel contaminated by the noise added at the receivers. A quasi-static fading or block-fading strategy is adopted here, where we keep the fading gain fixed for the whole duration of a transmission frame of the same link, which represents slow-fading channels. A modification of the SINR utilised in AWGN is necessary for formulating the SINR in Rayleigh fading channels, which is given by Goldsmith and Chua [14] $\widetilde{\Gamma}_{l_{i, j}, n}=\left(\left(H_{i, j} G_{i, j} P_{i, n}\right) /\right.$ $\left.\left(\sum_{i^{\prime} \neq i} H_{i^{\prime}, j} G_{i^{\prime}, j} P_{i^{\prime}, n}+N_{0}\right)\right)$, where $H_{i, j}=\left|h_{i, j}\right|^{2}$ denotes the fading gain of the link between the transmitter $i$ and receiver $j$. Note that the SINR of each link in the network

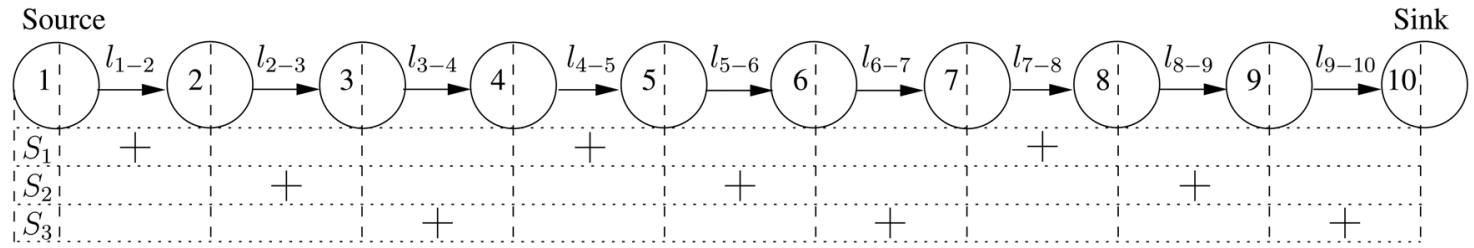

Fig. 1 String topology, where the $S N$ and the DN is linearly connected by intermediate nodes relying on link scheduling associated with the SPTS parameter of $T=3$, when $N=3$ and $V=10$ 
265 cannot be lower than the target SINR required for maintaining the target QoS, where the NL and the transmit power variables may vary.

We rely on the spatially periodic time sharing (SPTS) technique of [7] for modelling periodic

270 transmit-TS-activation-scheduling, where we consider a distance of $T$ between pairs of nodes, which are transmitting in the same TS. The same TSs are reactivated after every $T$ TSs. Fig. 1 illustrates the SPTS for $T=3$, where $\left[S_{1}, S_{2}, \ldots, S_{N}\right]$ describes each TS and + denotes the 275 active links. For example, during the first TS, links $l_{1,2}, l_{4,5}$, $l_{7,8}$ are scheduled for simultaneous transmissions, which only moderately interfere with each other and each link is activated only once during the whole TDMA frame. For simplicity in our system model, we use $T=3$ in our 280 SPTS-aided interference-limited scenario.

We model the physical layer for the sake of characterising the upper layers with the aid of a BER-SNR look-up table (LUT) relationship for the system model considered, which provides us with the SNR value required for maintaining a

285 given target BER. Explicitly, in this contribution we consider an uncoded BPSK modulated system and a 1/2-rate convolutional coded (CC) hard-decoded [Hard

Q3 decoding compares the individual bits of the received bit sequence with all possible codewords with the aid of the

290 Hamming distance approach.] [15] as well as soft-decoded [Soft decoding compares the received codeword with al possible codewords with the aid of the minimum Euclidean distance approach in order to attain a measure of confidence in terms of probabilities.] [15] QPSK scheme communicating

295 over an AWGN channel.

We also consider the BER-SNR LUTs of our iterative decoding aided system, as shown in Fig. 2 for 1/2-rate serially concatenated codes (SCCs) using 1, 5 and 10 decoding iterations, where the first iteration corresponds to the 1/2-rate-CC-Soft QPSK MCS [16]. We

300 can determine the NL of any of the different

MCSs by relying on their BER-SNR LUT for the system considered. Note that we invoke a decode and forward (DF) scheme in two different scenarios. In the first scenario, we neglect the ED of the coding and decoding operations,

305 which we refer to as Scenario 1 in our forthcoming analysis. On the other hand, in the second scenario, we include both the coding and decoding ED in our analysis, which we refer to as Scenario 2. Therefore, Scenario 1 constitutes a benchmark of the NL, since it only considers

310 the transmit power, hence giving us the best possible NL we can achieve. However, Scenario 2 is a more realistic one, where we additionally consider the SPP. Therefore we can evaluate the NL difference between the best case and

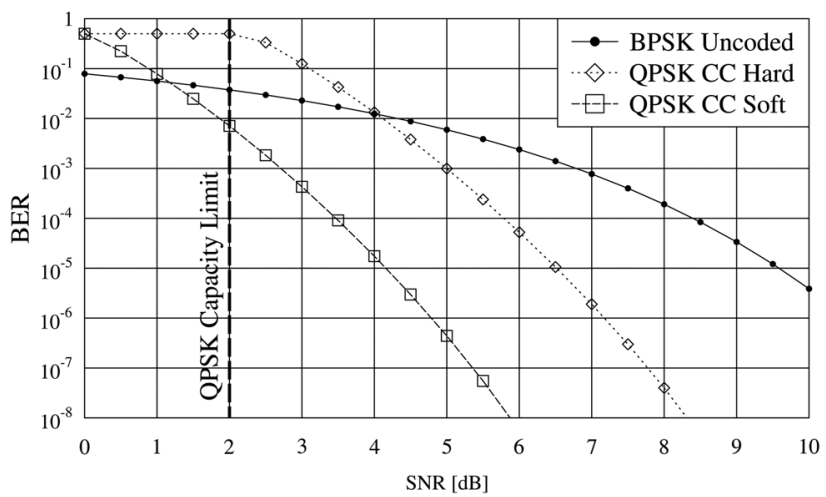

Fig. 2 BER versus $S N R[d B]$ for an $A W G N$ channel

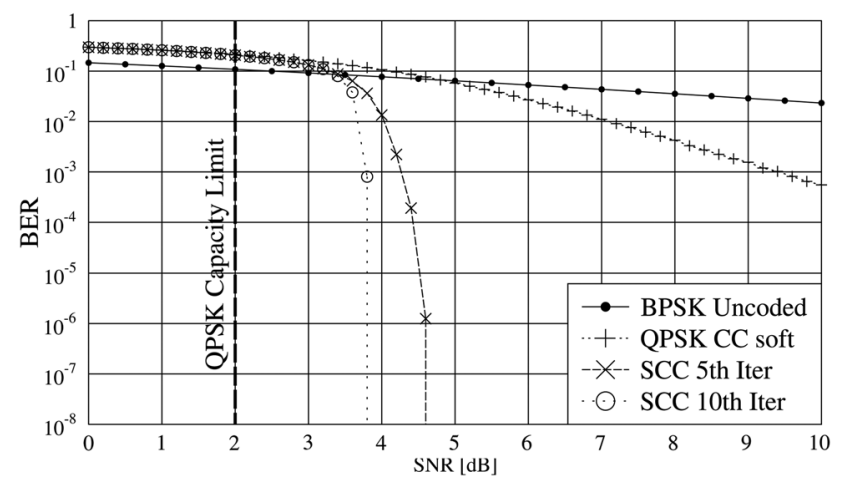

335

Fig. 3 BER versus $S N R[d B]$ for an independently block Rayleigh fading channel

the realistic scenarios. The SPP is modelled by the signal processing (SP) ED considered in [12]. At the DN, all ED is ignored, since we assume that the DN is plugged into the mains power source. The action of the BER-SNR LUT of the system model considered for both the AWGN and Rayleigh fading channels can be observed in Figs. 3 and 4, respectively.

\section{$2.3 N$ N model}

The NL may be interpreted differently, depending on the specific application, as mentioned in [17-20]. For example, the NL may be maximised by incorporating energy awareness into each individual node [21]. Moreover, an optimal hop-length was obtained by the simultaneous computation of the optimal transmission power, the optimal SNR and the optimal BER in a Rayleigh fading environment in [22]. Specifically, Zhang and Gorce [22] demonstrated that in a relay-aided scheme, optimising the hop-length is capable of significantly extending the NL. In our study, $T_{i}$ is the lifetime of an individual node in the network, which corresponds to the time instant, when node $i$ has completely discharged its battery. The NL is then defined as the earliest time instant, at which any of the nodes drains its battery, which is given by $T_{\text {net }}=\min _{i \neq V, i \in \mathcal{V}} T_{i}$.

\section{Problem formulation}

Having described the system model in Section 2, the NL maximisation problem [7] can be formulated as in (1)-(4). The links that are active in TS $n$ are denoted by the set $\mathcal{L}_{n}$. The specific variables of the optimisation problem are $T_{\text {net }}$ and $P_{l, n}$ for $l \in \mathcal{L}_{n}, n=1, \ldots, N$, where we have $N=3$ TSs, say $l_{1,2}, l_{4,5}$ and $l_{7,8}$, per link. We assume that any operation other than the transmission and SP in the network incurs a negligible ED. The power amplifier's efficiency is denoted by $\alpha$ [23], while $l \in \mathcal{O}(i)$ and $l \in \mathcal{I}(i)$ represent the

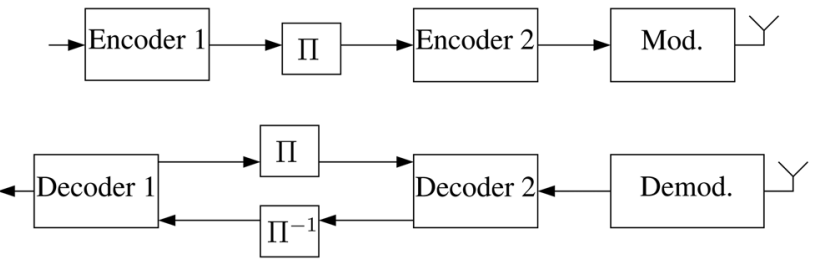

Fig. 4 Block diagram of the iteratively decoded system 
transmit link and the receive link of node $i$, respectively. Furthermore, $\left\{i: i \in \mathcal{O}^{-1}(l), l \in \mathcal{L}_{n}\right\}$ in (6) describes the set of nodes, where the transmit links are connected and activated in the same TS

$$
\begin{array}{lrl} 
& \max \quad T_{\text {net }} \\
\text { s.t. } & \Gamma_{l_{i, j}, n} \geq \gamma, \quad \forall n, l_{i, j} \in \mathcal{L}_{n}
\end{array}
$$

405

$$
\frac{T_{\text {net }}}{N} \sum_{n=1}^{N}\left(\sum_{l \in \mathcal{O}(i) \cap \mathcal{L}_{n}}\left((1+(1-\alpha)) \cdot P_{i, n}+P_{\mathrm{sp}}\right)\right) \preceq \mathcal{E}_{i}, \quad \forall i
$$

$$
\begin{gathered}
0 \preceq P_{i, n} \preceq\left(P_{i}\right)_{\max }, \quad \forall n, i \\
\min q \\
\text { s.t. } \quad \gamma \cdot\left(\sum_{i^{\prime} \neq i} G_{i^{\prime}, j} \cdot P_{i^{\prime}, n}+N_{0}\right)-G_{i, j} \cdot P_{i, n} \leq 0 \\
\quad \forall n, \quad\left\{i: i \in \mathcal{O}^{-1}(l), l \in \mathcal{L}_{n}\right\} \\
\sum_{n=1}^{N}\left(\sum_{l \in \mathcal{O}(i) \cap \mathcal{L}_{n}}\left((1+(1-\alpha)) \cdot P_{i, n}+P_{\mathrm{sp}}\right)\right)-q \cdot \mathcal{E}_{i} \cdot N \leq 0, \quad \forall i \\
0 \leq P_{i, n} \leq\left(P_{i}\right)_{\max }, \quad \forall n, i
\end{gathered}
$$

We can readily transform the NL maximisation problem into the minimisation of the reciprocal of the NL, which is formulated as $q=\left(1 / T_{\text {net }}\right)$ by using a change of variable in order to avoid multiplication of the pair of optimisation variables, which is generally non-convex, as exemplified in (3). The energy conservation constraint of each node is formulated in (3), which represents how the initial battery energy, $\mathcal{E}_{i}$ can be dissipated as a function of the system parameters, until the battery is discharged. Therefore, to transform the optimisation problem into a convex one, both (1) and (3) are rewritten as (5) and (7), respectively, while (2) and (6) represent the lower bound of the signal quality required for each link with a range of target SINR values, which are denoted by $\gamma$. The maximum affordable transmit power of a node is given by (8).

Our optimisation problem is solved with the aid of the interior point method described in [24], which can be used for solving both linear and non-linear convex optimisation problems for each link of a spatially periodic link schedule defined by the specific SPTS considered for the string network topology of Fig. 1.

\section{Experimental results}

In this section, we analyse the NL of both Scenarios 1 and 2 against the attainable BER performances for different MCSs under both AWGN and Rayleigh fading channels. We compute two types of BER performances. The first one is the per-link BER requirement (PLBR), which defines the BER value attained by the weakest link. This represents the worst BER that can be tolerated over the links. In other words, the BER of each link must be lower than the PLBR. By contrast, the end-to-end BER (E2EB) is defined as the BER accumulated along the route spanning from the $\mathrm{SN}$ to the DN, which is calculated as $\mathrm{BER}_{\text {end }}=1-\prod_{l=1}^{V-1}$ $\left(1-\mathrm{BER}_{l}\right)$, where $\mathrm{BER}_{l}$ is a function of the SINR $\left(\mathrm{BER}_{l}\right.$ $\left.=f_{\mathrm{MCS}}\left[\mathrm{SINR}_{l}\right]\right)$ that may be fetched from the LUT selected
Table 1 System parameters used in our simulations

\begin{tabular}{lc}
\hline Simulation parameter & Value \\
\hline path loss exponent, $m$ & 3 \\
geographic distance between consecutive nodes, $d, \mathrm{~m}$ & 30 \\
noise power, $N_{0}, \mathrm{dBm}$ & -60 \\
initial energy per node, $\mathcal{E}_{i}, \mathrm{~J}$ & 5000 \\
maximum affordable transmit power of a node, & $10[25]$ \\
$\left(P_{i}\right)_{\text {max }}, \mathrm{mW}$ & \\
number of slots per frame, $N$ & 3 \\
number of nodes, $V$ & 10 \\
number of links, $V-1$ & 9 \\
efficiency of the power amplifier, $\alpha$ & 0.6 \\
SPTS parameter, $T$ & {$[23]$} \\
\hline
\end{tabular}

for the specified MCS and $(V-1)$ is the number of links along the route. Each target SINR returns $(V-1)$ number of actual link SINRs and each link SINR corresponds to a BER value read from the given LUT. Finally, the $(V-1)$ BERs provide us with a single accumulated E2EB. A PLBR, an E2EB and a single NL value are hence computed for each corresponding target SINR. The experimental results are for 'continuous-time' NL, which corresponds to the NL obtained by continuous transmission.

For our simulations, we use the system parameters of Table 1 for both the AWGN and Rayleigh fading channels of Scenarios 1 and 2. The initial battery energy per node is $\mathcal{E}_{i}=5000 \mathrm{~J}$, which corresponds to the capacity of an AAA alkaline long-life battery. In addition, the SPP utilised in Scenario 2 is $P_{\mathrm{sp}}=0.44 \mathrm{~mW}$ [12] and the number of simulation trials used for the fading scenario is $\tau_{\text {fading }}=2000$.

\subsection{NL against BER for an AWGN channel}

We present our NL results for Scenarios 1 and 2 against the PLBR and E2EB [In all figures, BER values higher than $10^{-3}$ are deemed to be inadequate for practical application scenarios. They are only illustrated for the sake of highlighting the explicit trade-offs between the NL and the BER performances.] for uncoded BPSK, as well as for hard-decoded and soft-decoded 1/2-rate CC QPSK MCSs for an AWGN channel in Figs. 5 and 6, respectively. Again, in Scenario 1 we first neglect the SPP and then include it in the analysis of Scenario 2, which we refer to by using the notation of SPP-X in Figs. 5 and 6. These figures show that as expected, the NL decays, when

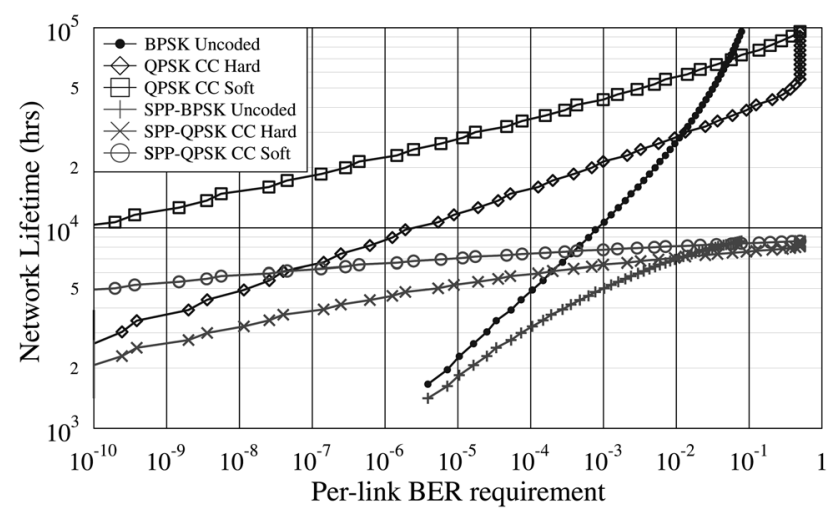

Fig. $5 N L$ for Scenarios 1 and 2 against PLBR for uncoded BPSK as well as for both hard-decoded and soft-decoded 1/2-rate CC QPSK MCSs under AWGN channel 


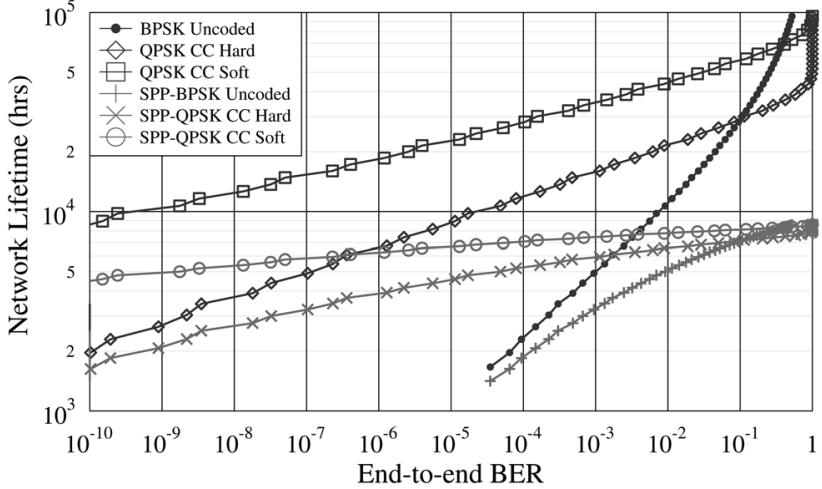

Fig. $6 N L$ for Scenarios 1 and 2 against E2EB for uncoded BPSK as well as for both hard-decoded and soft-decoded 1/2-rate CC QPSK MCSs under AWGN channel

requiring a reduced target BER, since the transmit power must be increased to achieve the target BER and vice versa. Having illustrated the trade-off between the NL and both the PLBR and the E2EB in Figs. 5 and 6, respectively, the designer can decide upon the optimal choice of MCS for maximising the NL. In Scenario 1, when aiming for a target BER of $10^{-3}$, the best NL is achieved by the soft-decoded 1/2-rate CC QPSK MCS, which is about $43773 \mathrm{~h}=4.99 \mathrm{yr}$ in terms of the PLBR and $35140 \mathrm{~h}=4 \mathrm{yr}$ in terms of the E2EB for an AWGN channel. On the other hand, considering the more realistic Scenario 2, and hence including the SPP in the analysis, the achievable NL is reduced to $0.89 \mathrm{yr}$ for PLBR and to $0.85 \mathrm{yr}$ for E2EB. The lowest NL for an AWGN channel and a PLBR of $10^{-3}$ is attained by the uncoded BPSK in Scenario 2, which is about $0.57 \mathrm{yr}$, and it increases to $1.2 \mathrm{yr}$ of $\mathrm{NL}$, where we only consider the transmit power in Scenario 1.

\subsection{NL against BER for Rayleigh fading channels and the maximum achievable $N L$}

Let us now consider the NL of Scenarios 1 and 2 against the

P2EB for uncoded BPSK, as well as the upper bound of the NL for soft decoded 1/2-rate CC, and 1/2-rate SCC [16] using 5 and 10 decoding iterations in conjunction with QPSK MCSs for Rayleigh fading channels in Figs. 7 and 8, respectively. Upon comparing Figs. 7 and 8 for the

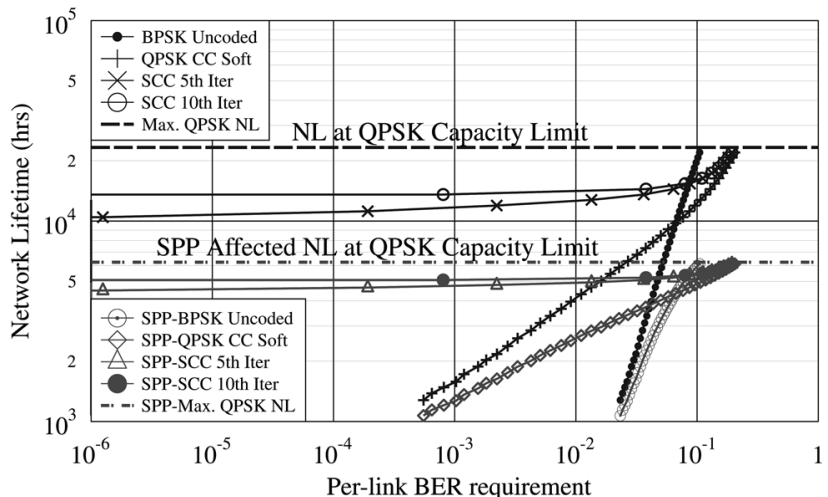

Fig. 7 NL for Scenarios 1 and 2 against PLBR for uncoded BPSK and the maximum achievable NL for soft decoded 1/2-rate CC QPSK and 1/2-rate SCC using 5 and 10 decoding iterations with QPSK for a Rayleigh fading channel

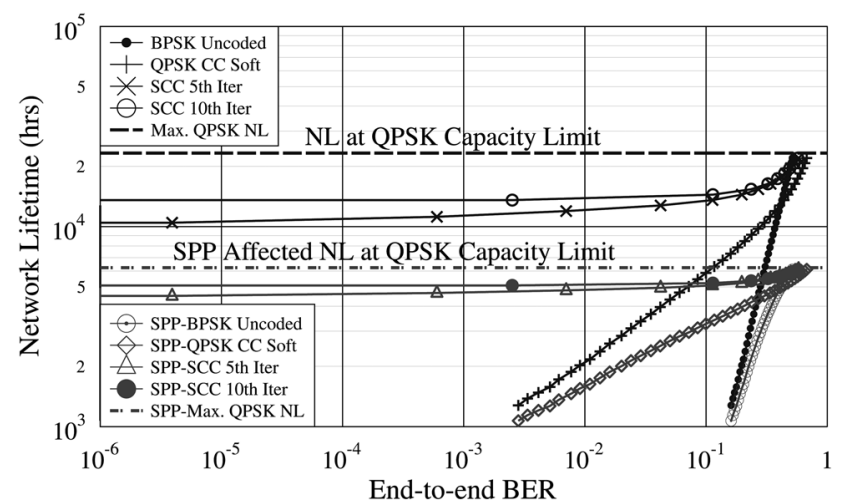

Fig. 8 NL for Scenarios 1 and 2 against E2EB for uncoded BPSK and the maximum achievable NL for soft decoded 1/2-rate CC QPSK and 1/2-rate SCC using 5 and 10 decoding iterations with QPSK for a Rayleigh fading channel

same NL values, the E2EB is seen to be substantially higher than the PLBR because of the bit errors accumulated, while relaying the information bits over intermediate nodes all the way, through to the DN. For a target BER of $10^{-3}$, the best NL for Scenario 1 is achieved by $1 / 2$-rate SCC using 10 decoding iterations for QPSK MCS, which is about $1.55 \mathrm{yr}$ in terms of the PLBR under Rayleigh fading channels, whereas upon considering the SPP of Scenario 2 about $0.58 \mathrm{yr}$ of NL is achieved for the same PLBR. The maximum NL that can be achieved is approximately $2.66 \mathrm{yr}$ in Scenario 1, while in Scenario 2, the maximum achievable NL is reduced to $0.71 \mathrm{yr}$, which is computed according to the SINR that corresponds to the capacity limit of the QPSK system considered.

\subsection{Discussions}

Again, the results of Figs. 5-8 illustrated that the NL is reduced upon reducing the target BER, which is owing to the increased transmit power required. More explicitly, the NL becomes undesirably short for low target BER values, which is because of the increased transmit power required to satisfy the target BER. As expected, the NL is longer for an AWGN channel than for a Rayleigh fading channel for the same SINR values and configurations because of the higher ED required for combating the deleterious effects of fading. Hence, provided that the application considered is capable of tolerating a higher BER, the NL becomes higher because of requiring a lower SINR value. Furthermore, the NL is substantially reduced because of the accumulated ED of SPP and transmit power. In other words, when considering higher target BER values requiring lower SINRs, the transmit power becomes negligible compared to the SPP. This is why there is a substantial difference between the NLs of Scenarios 1 and 2 at higher BER values. On the other hand, when considering lower target BER values, the required transmit power increases and it becomes more dominant than the SPP in the analysis and this is why the difference between the NLs of Scenarios 1 and 2 reduces, as the BER reduces. Nonetheless, when aiming for achieving the same target PLBR and E2EB, the $\mathrm{NL}$ is always shorter for E2EB because of the higher SINR required, which necessitates a higher transmit power. It is observed that for an AWGN channel and for both a PLBR as well as for an E2EB of $10^{-3}, 1 / 2$-rate CC soft-decoded QPSK doubles the NL of its hard-decoded counterpart, 
Table 2 'Continuous-time' NL (in years) evaluation for WSNs with given configurations

\begin{tabular}{|c|c|c|c|c|c|c|c|c|c|}
\hline & \multicolumn{2}{|c|}{$10^{-4}$ PLBR } & \multicolumn{2}{|c|}{$10^{-4}$ E2EB } & \multicolumn{2}{|c|}{$10^{-3}$ PLBR } & \multicolumn{2}{|c|}{$10^{-3}$ E2EB } & \\
\hline & $\operatorname{Scn} 2$ & Scn 1 & Scn 2 & Scn 1 & Scn 2 & Scn 1 & Scn 2 & Sen 1 & \\
\hline uncoded-BPSK & 0.36 & 0.55 & 0.21 & 0.27 & 0.57 & 1.2 & 0.37 & 0.57 & AWGN \\
\hline \multirow[t]{3}{*}{ QPSK-CC-Soft } & 0.85 & 3.97 & 0.80 & 3.20 & 0.89 & 4.99 & 0.85 & 4.00 & \\
\hline & \multicolumn{2}{|c|}{$10^{-2}$ PLBR } & \multicolumn{2}{|c|}{$10^{-2}$ E2EB } & \multicolumn{2}{|c|}{$10^{-3}$ PLBR } & \multicolumn{2}{|c|}{$10^{-3}$ E2EB } & \\
\hline & Scn 2 & Scn 1 & Scn 2 & Scn 1 & $\operatorname{Sen} 2$ & Scn 1 & Scn 2 & Scn 1 & \\
\hline QPSK-CC-Soft & 0.30 & 0.47 & 0.18 & 0.24 & 0.14 & 0.18 & - & - & fading \\
\hline SCC-10th-Iter & 0.58 & 1.57 & 0.58 & 1.55 & 0.58 & 1.55 & - & - & \\
\hline
\end{tabular}

when we ignore the SPP. Similarly, it has an up to four times longer NL than uncoded BPSK for an AWGN channel and a PLBR of $10^{-3}$, when only considering the transmit power. On the other hand, when the more realistic scenario of including the SPP in the analysis is considered, the 1/2-rate CC soft-decoded QPSK has a factor of 1.2 higher NL than its hard-decoded counterpart at a PLBR and E2EB of $10^{-3}$, and 2.3 times more NL for a E2EB of $10^{-3}$ than uncoded BPSK.

Table 2 presents the 'continuous-time' NL, in years for different MCSs and different target BER values in terms of both the PLBR and E2EB, and provides an overview of the systems, indicating both the longest and the shortest NL in Scenarios 1 and 2 for both AWGN and fading channels, where 'scenario' is abbreviated by 'Scn'. Therefore, the designer can readily determine, which systems and configurations are more NL-aware for a realistic application. For example, observe in Table 2 that the longest NL is achieved for an AWGN channel by soft decoded 1/2-rate CC for a PLBR of $10^{-4}$ in Scenario 1 , which is nearly $3.97 \mathrm{yr}$. By contrast, the lowest NL for an AWGN channel is accomplished by uncoded BPSK at an E2EB of $10^{-4}$ in Scenario 2, which is about 0.21 yr. Uncoded BPSK results in an even lower NL in a Rayleigh fading scenario, but the exact PLBR and E2EB values cannot be provided, since this would require an excessive transmit power. The longest NL is achieved for Rayleigh fading channels by 1/2-rate SCC using 10 decoding iterations at a PLBR of $10^{-2}$ in Scenario 1, which corresponds to $\mathrm{NL}=1.57 \mathrm{yr}$. Nonetheless, up to $4.99 \mathrm{yr}$ of NL can be accomplished by soft decoded 1/2-rate CC for a PLBR of $10^{-3}$ for AWGN channels in Scenario 1, which is reduced to $0.18 \mathrm{yr}$ for Rayleigh fading channels.

In this treatise, we consider two realistic applications. In Application 1, the environmental sensor network of [26] is considered, where we monitor the behaviour of ice caps and glaciers to deeply understand the changes in the Earth's climate in AWGN channel conditions. We assume that the $\mathrm{SN}$ transmits a data packet to the DN only once per day by activating 3 TSs in our $T=3$ SPTS. Therefore Fig. 1 shows that the data has to be stored at the respective node at the end of the day after activating 3 TSs per day. On the third day, the data packet arrives at the DN. In Application 2, we consider placing the sensors on linearly-spaced non-LOS lamp posts, which are $30 \mathrm{~m}$ apart from each other in order to quantify the level of pollution in the context of urban Rayleigh fading channels, where the channel is activated 12 times a day, which corresponds to a duty-cycle of $1 \mathrm{~s}(\mathrm{sec})$ of every $2 \mathrm{~h}$ for the sake of a fair evaluation of the pollution at different times of a day. The duration of each while achieving a 1.5 times higher NL for a PLBR of $10^{-3}$
TS is assumed to be $1 \mathrm{~s}$ in both of these application scenarios as well as in the optimisation problem. Application 1 of the system advocated in an environmental WSN at a PLBR of $10^{-3}$ has a maximum NL of $143911 \mathrm{yr}$ [The longest NL of $143911 \mathrm{yr}$ is the NL of the considered system, where each node transmits for $1 \mathrm{~s}$ every three days and this corresponds to $43773 \mathrm{~h}=4.99 \mathrm{yr}$ of continuous transmission.], which is achieved by $1 / 2$-rate CC reduced to $25540 \mathrm{yr}$ [The $25540 \mathrm{yr}$ of NL corresponds to $7768 \mathrm{~h}=0.89 \mathrm{yr}$ of continuous transmission.] because of the SPP. On the other hand, in Application 2, up to $3716 \mathrm{yr}$ of NL is accomplished by 1/2-rate SCC using 10 decoding iterations at a PLBR of $10^{-3}$ and the NL reduced to $1392 \mathrm{yr}$ because of SPP in a Rayleigh fading channel. Our results illustrated that the NL is strictly dependent on the specific choice of the physical layer parameters, such as the MCSs, transmit power, target BER and target SNR as well as on the duty cycle and TS duration.

We have shown how the requirement of different target BER values at the physical layer affected both the NL and the network performance in the string topology considered. We also illustrated the dominant impact of the SPP on the NL compared to that of the transmission power. A useful further investigation would be to ascertain, what type of modulation schemes should be used for what type of networks or applications. Therefore, depending on the QoS requirements, the network designer can make beneficial decisions for specific systems, depending on the network performance required [We acknowledge the anonymous reviewer for his constructive comments.].

\section{Conclusions}

We evaluated the NL for both Scenario 1, where we consider the required transmit power only, and for Scenario 2, where we considered an additional SPP, against the BER target of WSNs for a fixed rate of different MCSs for both AWGN and Rayleigh fading channels. The NL maximisation problem was formulated with the aid of a set of lower bounded target SINR values for each link for characterising the BER-dependent QoS and the impact of the physical layer parameters on the NL in the string topology considered. We presented experimental results for assisting the network designer in making informed decisions as to which MCS works well for the application supported. The experimental results illustrated that 1/2-rate CC soft-decoded QPSK MCS provided the longest NL for an AWGN channel, which is nearly $4.99 \mathrm{yr}$ of 'continuous-time' $\mathrm{NL}$ at $10^{-3}$ of PLBR in Scenario 1. On the other hand, SPP soft-decoded QPSK for an AWGN channel and the NL is 
has a substantial impact on the NL, which reduced the NL to $0.89 \mathrm{yr}$ in Scenario 2. Two realistic applications were 795 considered. In the first application having a transmission duty-cycle of $1 \mathrm{~s}$ per day, a NL of $143911 \mathrm{yr}$ is achieved by $1 / 2$-rate CC soft-decoded QPSK at a PLBR of $10^{-3}$ for an AWGN channel, which is reduced to $25540 \mathrm{yr}$ because of SPP. In the second application having a transmission

800 duty-cycle of $1 \mathrm{~s}$ of every $2 \mathrm{~h}$, a NL of $3716 \mathrm{yr}$ is accomplished by 1/2-rate SCC using 10 decoding iterations at a PLBR of $10^{-3}$, which is reduced to $1392 \mathrm{yr}$ because of SPP in Rayleigh fading channels.

\section{Acknowledgments}

This research has been funded by the Republic of Turkey Ministry of National Education, and the Industrial Companies who are Members of the Mobile VCE, with 810 additional financial support from the UK Government's Engineering \& Physical Sciences Research Council (EPSRC). The financial support of the Research Councils UK (RCUK) under the India-UK Advanced Technology Center (IU-ATC), of the EU under the auspices of the 815 Concerto project, and of the European Research Council's Senior Research Fellow Grant is also gratefully acknowledged.

\section{References}

1 Cheng, C.-M., Kung, H.T.: 'Use of relays in extending network lifetime'. IEEE 60th Vehicular Technology Conf. (VTC'04), Los Angeles, CA, USA, September 2004

2 Duan, D., Qu, F., Yang, L., Swami, A., Principe, J.: 'Modulation selection from a battery power efficiency perspective: a case study of PPM and OOK'. IEEE Wireless Communications and Networking Conf. (WCNC'09), Budapest, Hungary, April 2009

3 Rosas, F., Oberli, C.: 'Modulation and SNR optimization for achieving energy-efficient communications over short-range fading channels', IEEE Trans. Wirel. Commun., 2012, 11, (12), pp. 4286-4295

4 Cui, S., Goldsmith, A., Bahai, A.: 'Energy-constrained modulation optimization', IEEE Trans. Wirel. Commun., 2005, 4, (5), pp. 2349-2360

5 Hartwell, J., Messier, G., Davies, R.: 'Optimizing physical layer energy consumption for wireless sensor networks'. IEEE 65th Vehicular Technology Conf. (VTC’07), Dublin, Ireland, April 2007

6 Sklar, B.: 'Digital communications: fundamentals and applications' (Prentice-Hall, 2001, 2nd edn.)

7 Madan, R., Cui, S., Lall, S., Goldsmith, A.: 'Cross-layer design for lifetime maximization in interference-limited wireless sensor networks', IEEE Trans. Wirel. Commun., 2006, 5, (11), pp. 3142-3152

8 Panigrahi, B., Sharma, A., De, S.: 'Interference aware power controlled forwarding for lifetime maximisation of wireless ad hoc networks', IET
Wirel. Sens. Syst., 2012, 2, pp. 22-30. Available at: http://www.digitallibrary.theiet.org/content/journals/10.1049/iet-wss.2011.0094

9 Chen, Y., Yang, Y., Yi, W.: 'A cooperative routing algorithm for lifetime maximization in wireless sensor networks'. IET Int. Conf. on Wireless Sensor Network, January 2010, pp. 167-172. Available at: http://www.digital-library.theiet.org/content/conferences/10.1049/cp. 2010.1047

10 Dahnil, D., Singh, Y., Ho, C.: 'Topology-controlled adaptive clustering for uniformity and increased lifetime in wireless sensor networks', IET Wirel. Sens. Syst., 2012, 2, pp. 318-327(9). Available at: http://www. digital-library.theiet.org/content/journals/10.1049/iet-wss.2012.0034

11 Agarwal, D., Kishor, N.: 'Network lifetime enhanced tri-level clustering and routing protocol for monitoring of offshore wind farms', IET Wirel. Sens. Syst., 2014, 4, pp. 69-79. Available at: http://www.digital-library. theiet.org/content/journals/10.1049/iet-wss.2013.0050

12 Li, L., Maunder, R., Al-Hashimi, B., Zwolinski, M., Hanzo, L. 'Energy-conscious turbo decoder design: a joint signal processing and transmit energy reduction approach', IEEE Trans. Veh. Technol., 2013, 62, (8), pp. 3627-3638

13 Goldsmith, A.: 'Wireless communications' (Cambridge University Press, 2005)

14 Goldsmith, A., Chua, S.-G.: 'Variable-rate variable-power MQAM for fading channels', IEEE Trans. Commun., 1997, 45, (10), pp. 1218-1230

15 Hanzo, L., Liew, T., Yeap, B.: 'Turbo coding, turbo equalisation and space-time coding' (John Wiley \& Sons, 2002). Available at: http:// www.eprints.soton.ac.uk/258252/

16 El-Hajjar, M., Hanzo, L.: 'Exit charts for system design and analysis', IEEE Commun. Surv. Tutorials, 2013, (99), pp. 1-27. Available at: http://www.eprints.soton.ac.uk/352320/

17 Al-Agha, K., Bertin, M.-H., Dang, T., et al.: 'Which wireless technology for industrial wireless sensor networks? The development of OCARI technology', IEEE Trans. Ind. Electron., 2009, 56, (10), pp. 4266-4278

18 Chen, Y., Zhao, Q.: 'On the lifetime of wireless sensor networks', IEEE Commun. Lett., 2005, 9, (11), pp. 976-978

19 Cheng, Z., Perillo, M., Heinzelman, W.: 'General network lifetime and cost models for evaluating sensor network deployment strategies', IEEE Trans. Mob. Comput., 2008, 7, (4), pp. 484-497

20 Chang, J.-H., Tassiulas, L.: 'Maximum lifetime routing in wireless sensor networks', IEEE/ACM Trans. Netw., 2004, 12, (4), pp. 609-619

21 Raghunathan, V., Schurgers, C., Park, S., Srivastava, M.: 'Energy-aware wireless microsensor networks', IEEE Signal Process. Mag., 2002, 19, (2), pp. 40-50

22 Zhang, R., Gorce, J.-M.: 'Optimal transmission range for minimum energy consumption in wireless sensor networks'. IEEE Wireless Communications and Networking Conf. (WCNC'08), Las Vegas, NV, USA, March 2008

23 Albulet, M.: 'RF power amplifiers' (SciTech, Raleigh, NC, 2001)

24 Byrd, R.H., Hribar, M.E., Nocedal, J.: 'An interior point algorithm for large-scale nonlinear programming', SIAM J. Optim., 1999, 9, (4), pp. $877-900$

25 IEEE Standard for Information Technology - Local and Metropolitan Area Networks - Specific Requirements - Part 15.4: Wireless Medium Access Control (MAC) and Physical Layer (PHY) Specifications for Low Rate Wireless Personal Area Networks (WPANs), Std. September 2006

26 Martinez, K., Hart, J., Ong, R.: 'Environmental sensor networks', IEEE Comput., 2004, 37, (8), pp. 50-56. Available at: http://www.eprints. soton.ac.uk/259997/ 


\section{www.ietdl.org}

${ }^{925}$ WSSSI20140049

Author Queries

Halil Yetgin, Kent Tsz Kan Cheung, Mohammed El-Hajjar, Lajos Hanzo

930 Q1 Please check and confirm the email address of the corresponding author.

Q2 Abstract should not exceed more than 200 words as per the style. Please check and amend.

Q3 All footnotes have been moved to text as required by journal style. Please check and confirm that they have been located correctly within the text. We have changed the figure citations [Fig. 4 to Fig. 2, Figs. 2 and 3 to Figs. 3 and 4]. Please check and approve.

Q5 Please check the edit made in Table 2.

Q6 Please check Ref. [6].

Q7 Please provide volume number for Ref. [16]. 